\title{
Validación estructural de una vivienda de interés social mediante, estudios analítico-experimentales
}

\author{
Herrera M. ${ }^{1}$; Gómez C. ${ }^{2}$; Parra G. ${ }^{3}$; Arévalo D. ${ }^{4}$; Hernández L. ${ }^{5}$; Placencia P. ${ }^{6}$ \\ ${ }^{1}$ Ayudante de Investigación, Centro de Investigación de la Vivienda, Escuela Politécnica Nacional, \\ Facultad de Ingeniería Civil y Ambiental, Quito, Ecuador \\ e-mail: melisa.herrera@epn.edu.ec \\ ${ }^{2}$ Coordinador Técnico, Centro de Investigación de la Vivienda, Escuela Politécnica Nacional, \\ Facultad de Ingeniería Civil y Ambiental, Quito, Ecuador \\ e-mail: christian.gomez@epn.edu.ec \\ ${ }^{3}$ Analista, Centro de Investigación de la Vivienda, Escuela Politécnica Nacional, \\ Facultad de Ingeniería Civil y Ambiental, Quito, Ecuador \\ e-mail: klever.parra@epn.edu.ec \\ ${ }^{4}$ Especialista, Centro de Investigación de la Vivienda, Escuela Politécnica Nacional, \\ Facultad de Ingeniería Civil y Ambiental, Quito, Ecuador \\ e-mail: diego.arevalo@epn.edu.ec \\ ${ }^{5}$ Profesor Titular, Escuela Politécnica Nacional, Facultad de Ingeniería Civil y \\ Ambiental, Quito, Ecuador \\ e-mail: luis.hernandezr@epn.edu.ec \\ ${ }^{6}$ Profesor, Escuela Politécnica Nacional, Facultad de Ingeniería Civil y \\ Ambiental, Quito, Ecuador \\ e-mail:pptt30@gmail.com
}

Artículo recibido en julio de 2018; aprobado en septiembre de2018.

${ }^{2}$ Ingeniero Civil, MBA.

Autor para correspondencia: christian.gomez@epn.edu.ec

\author{
${ }^{3}$ Ingeniero Civil, mención Estructuras. \\ ${ }^{4}$ Ingeniero Mecánico, MSc. \\ ${ }^{5}$ Ingeniero Civil, $\mathrm{PhD}$ \\ ${ }^{6}$ Ingeniero Civil, MSc.
}

\section{Resumen}

En la presente investigación se validó estructuralmente una vivienda de interés social, la cual posee un sistema constructivo innovador de paneles tipo sándwich.

Para validar la vivienda se realizaron varios estudios experimentales que incluyen pruebas de campo y ensayos de laboratorio, tanto en los materiales como en el prototipo de vivienda. Para los ensayos se utilizaron las normas ASTM y el código ACI-318, bajo la dirección técnica del Centro de Investigación de la Vivienda de la Escuela Politécnica Nacional. De los estudios realizados se obtuvieron curvas de capacidad, rigidez, esfuerzos y módulos de elasticidad de los materiales, evidenciando que la vivienda presenta un buen comportamiento ante cargas verticales y laterales (acciones sísmicas). Adicionalmente se realizó un estudio analítico con la ayuda de un modelo matemático tridimensional para conocer la distribución de esfuerzos en la vivienda y compararlos con los esfuerzos obtenidos experimentalmente.

Los resultados obtenidos mostraron que la vivienda es apta para resistir solicitaciones gravitacionales y laterales que requiere la norma NEC-15.

Palabras clave: vivienda, paneles sándwich, innovación, poliestireno expandido, poliisocianurato.

Abstract

In the present investigation, a housing of social interest was validated structurally, which has an innovative constructive system of sandwich panels.

To validate the house, several experimental studies were carried out, including field tests and laboratory tests, both on the materials and on the housing prototype. For the tests, the ASTM standards and the ACI-318 code were used, under the technical direction of the Housing Research Center of the National Polytechnic School. From the studies carried out, curves of capacity, rigidity, stresses and modulus of elasticity of the materials were obtained, showing that the house presents a good behavior before vertical and lateral loads (seismic actions). Additionally, an analytical study was carried out with the help of a three-dimensional mathematical model to know the distribution of efforts in the house and compare them with the efforts obtained experimentally.

The obtained results showed that the house is apt to resist gravitational and lateral solicitations that the NEC-15 standard requires 
Keywords: housing, sandwich panels, innovation, expanded polystyrene, polyisocyanurate

\section{Introducción}

Ecuador ha sido constantemente escenario de varios eventos sísmicos y la destrucción que estos dejan a su paso no ha disminuido. El sismo del 16 de abril del 2016, permitió comprobar que la mayoría de las estructuras en el país son vulnerables dado que un buen número de estructuras son construidas de manera empírica y otras simplemente no tienen un control técnico y de calidad adecuado. Por tales razones es necesario investigar e implementar nuevos sistemas y métodos constructivos, además de optimizar los existentes. Estos nuevos sistemas estructurales deben ser resistentes, livianos, durables y de bajo costo y que cumplan con estándares sismo-resistentes.

Una empresa ecuatoriana, fabricó y construyó una vivienda de interés social con un sistema de paneles tipo sándwich, que se espera pueda ayudar en la reconstrucción de las poblaciones afectadas de Manabí, es por ello que busca validar estructuralmente la vivienda para que pueda ser una solución segura y funcional para la sociedad.

El sistema de paneles tipo sándwich, es un sistema constructivo no convencional que ofrece muchos beneficios tales como:

- Auto-portante.

- Aislante termo-acústico.

- Sostenibilidad en su fabricación.

- Ligereza.

- Facilidad y rapidez de montaje.

- Excelente relación peso-resistencia.

- Compatibilidad con otros sistemas constructivos.

- Acabados de alta calidad.

Iza en el 2012, menciona "La perfecta combinación entre la rigidez que proporciona la chapa metálica y las buenas propiedades de aislamiento de la espuma de poliuretano hacen que este material compuesto, bajo el denominado efecto sánduche, tenga una óptima resistencia acorde al tipo de solicitación de carga a la que esté expuesto, ya que por separado tanto la chapa metálica como el poliuretano no las alcanzaría (...)" [1, p. 200]. Abeysinghe, et al. en 2013 concluyen el sistema HCFPS (Sistema Hibrido Compuesto de Placa de Piso) se puede utilizar como una alternativa viable al sistema de piso convencional ya que cumple requisitos de rendimiento estructural y tiene muchas propiedades deseables [2]. Según Bournas et al. en el 2012, el estudio que realizaron sobre el sistema estructural de paneles tipo sándwich prefabricados de hormigón armado (RCSP) obtuvo resultados que muestran que es un sistema de construcción prometedor para regiones de sismicidad moderada y alta [3]

Al ser un sistema no convencional cuyos parámetros de diseño no están definidos en la Norma Ecuatoriana de la Construcción (NEC-15), esta debe ser estudiada, basándose en el apartado 2.3 del capítulo 9 de la norma NEC-15 (Viviendas de hasta 2 pisos con luces de hasta $5 \mathrm{~m}$ ), el cual indica que, "para sistemas constructivos diferentes a los descritos en este capítulo, cuyo diseño no pueda ser respaldado por normativa nacional o internacional o cuando se trate de un sistema único o patentado, éstos deberán ser aprobados por el Comité Ejecutivo de la Norma Ecuatoriana de la Construcción y contar con un informe técnico sobre el desempeño del sistema constructivo, y el cumplimiento de las disposiciones de la NEC, emitido por el Centro de Investigación de la Vivienda (CIV) de la Escuela Politécnica Nacional u otro centro certificado por el Comité Ejecutivo de la NEC" [4].

Para la validación estructural se realizan varios ensayos en los materiales con el fin de determinar sus propiedades físico-mecánicas. Y se realizan pruebas a carga vertical y carga lateral en la vivienda, para conocer su desempeño, adicionalmente se determina el periodo experimental de la estructura mediante un estudio de vibraciones ambientales. Como lo solicita la norma estos ensayos son ejecutados en laboratorio y en campo por el Centro de Investigación de la Vivienda de la Escuela Politécnica Nacional.

Con los resultados obtenidos de dichos ensayos se verifica si la vivienda cumple con requisitos de 
resistencia y rigidez para hacer frente a las cargas verticales y laterales cumpliendo con deflexiones y derivas establecidas en la NEC-15. Además, se realizan modelos matemáticos tridimensionales para luego de calibrarlos compararlos con los resultados experimentales obtenidos y verificar que la vivienda cumple con requisitos de esfuerzos máximos y otros parámetros de desempeño de los materiales.

\section{Materiales fuentes y métodos}

\subsection{Descripción de los paneles}

Los paneles tipo sándwich poseen un material aislante termo-acústico unido a dos láminas de $0.4 \mathrm{~mm}$ de acero estructural A36 producidos mediante un proceso continuo, que actúan monolíticamente para resistir esfuerzos exteriores. Un recubrimiento galvalume por inmersión en caliente, ofrece resistencia a los efectos de la intemperie [5].

\subsection{Descripción arquitectónica de la vivienda}

El modelo propuesto es una vivienda de $42 \mathrm{~m}^{2}$ de área de construcción, de una planta con cubierta a doble caída y con una distribución de paredes que permiten separar los espacios esenciales como se muestra en los gráficos 1 y 2 .

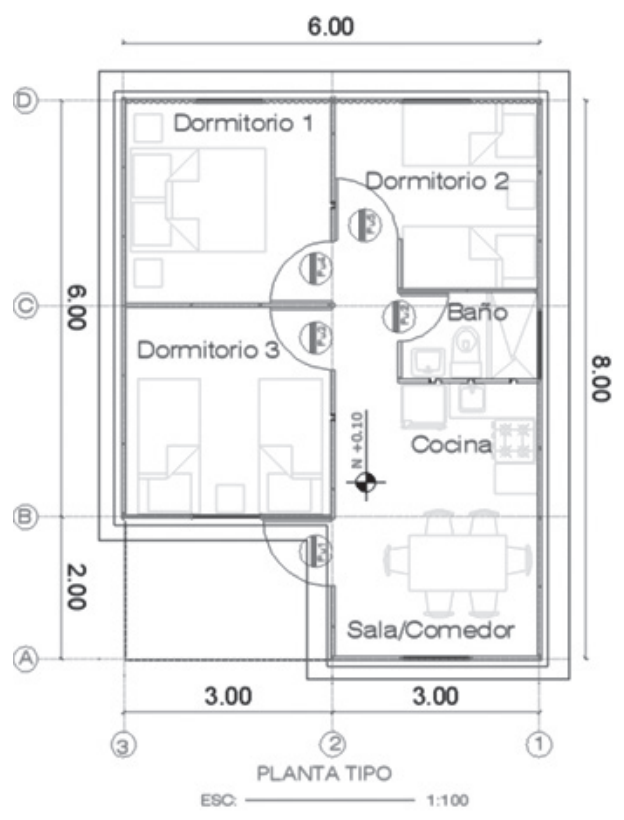

Gráfico 1. Plano arquitectónico de la vivienda.
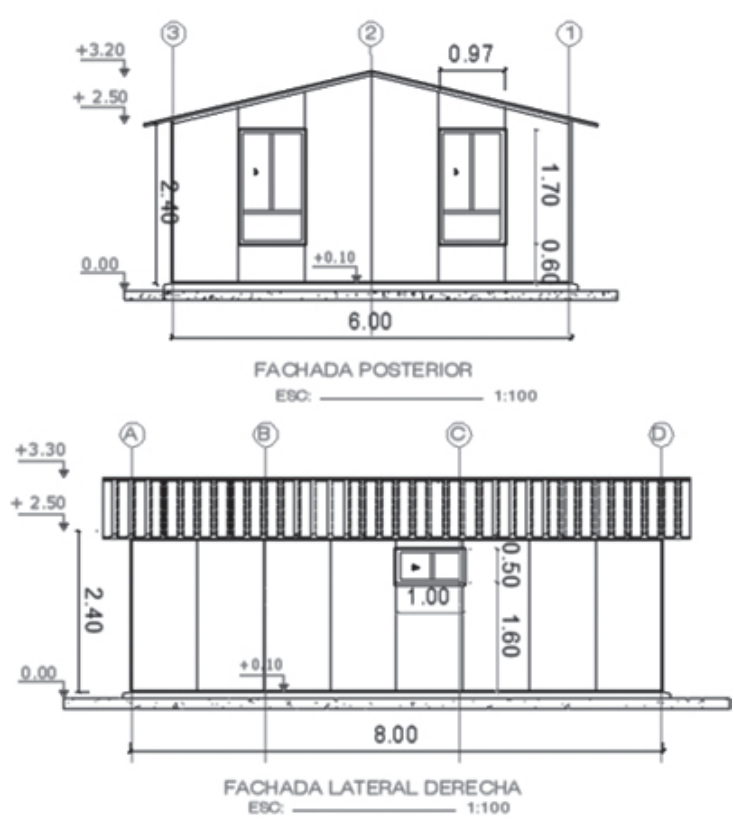

Gráfico 2. Plano arquitectónico de fachadas posterior y lateral dere-

cha.

\subsection{Descripción estructural de la vivienda}

La vivienda está basada en un conjunto de paredes auto-portantes y cubierta a dos aguas con una pendiente del $30 \%$. Las paredes son la estructura resistente y se encuentran sobre un perfil metálico tipo $\mathrm{C} 55 \times 30 \times 2$ unido a un contrapiso de hormigón de $20.6 \mathrm{MPa}\left(210 \mathrm{~kg} / \mathrm{cm}^{2}\right)$ mediante pernos de $10 \mathrm{~cm}$ de longitud (4") cada $50 \mathrm{~cm}$.

Las paredes y divisiones internas están conformadas por paneles de EPS (poliestireno expandido) de $50 \mathrm{~mm}$ de espesor, mientras que la cubierta está conformada por paneles de PIR (poliisocianuato) de $15 \mathrm{~mm}$ de espesor.

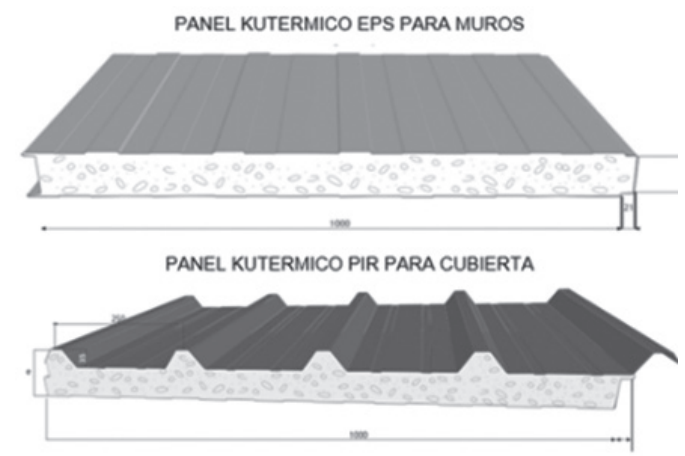

Gráfico 3. Paneles tipo sándwich para paredes y cubierta. 


\subsection{Estudios experimentales en materiales}

Se realizan diferentes ensayos para determinar algunas de las propiedades físico mecánicas de los materiales a utilizar en esta investigación, como son el poliestireno expandido (EPS), el poliisocianurato (PIR) y el acero estructural.

Ensayo de Densidad: El ensayo se realiza utilizando la norma ASTM C271 [6], y permite conocer la densidad y contenido de humedad de los elementos núcleo.

Ensayo de Corte: Este ensayo se realiza de acuerdo a la norma ASTM C393 [7] para determinar el esfuerzo máximo a corte del material del núcleo del panel.
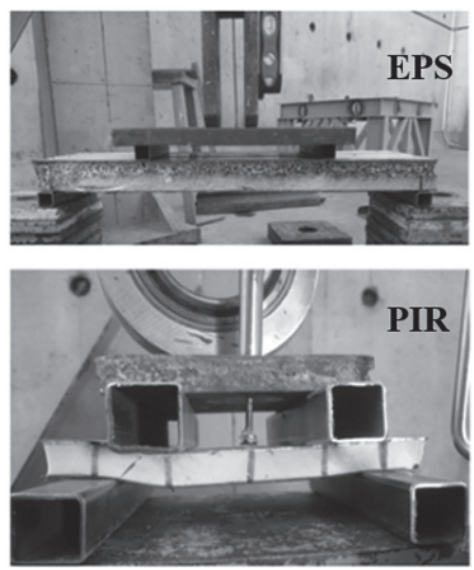

Gráfico 4. Ensayo de corte.

Ensayo a Compresión Perpendicular: Este ensayo se realiza utilizando la norma ASTM C365 [8] para determinar el esfuerzo a compresión perpendicular, y el módulo de elasticidad a compresión.
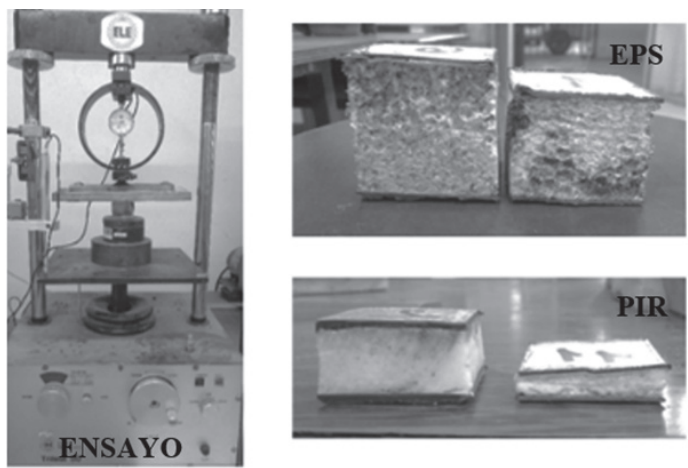

Gráfico 5. Ensayo de compresión perpendicular.
Para el ensayo se toman cinco muestras de cada material y se grafican los resultados esfuerzo y deformación unitaria para determinar el módulo de elasticidad con la pendiente de las curvas.

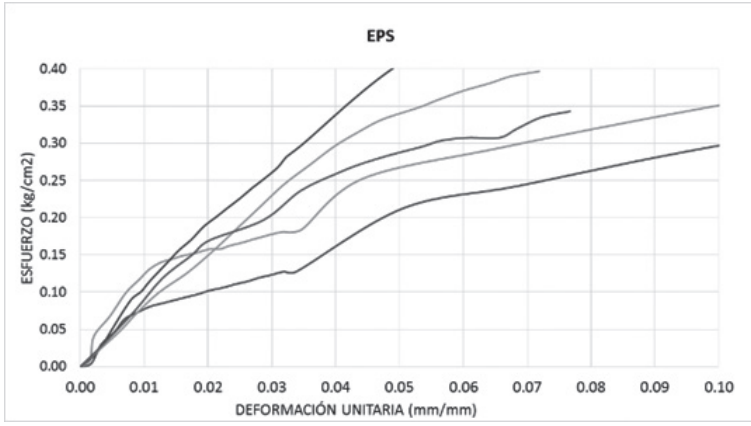

Gráfico 6. Curvas esfuerzo versus deformación unitaria en muestras de EPS.

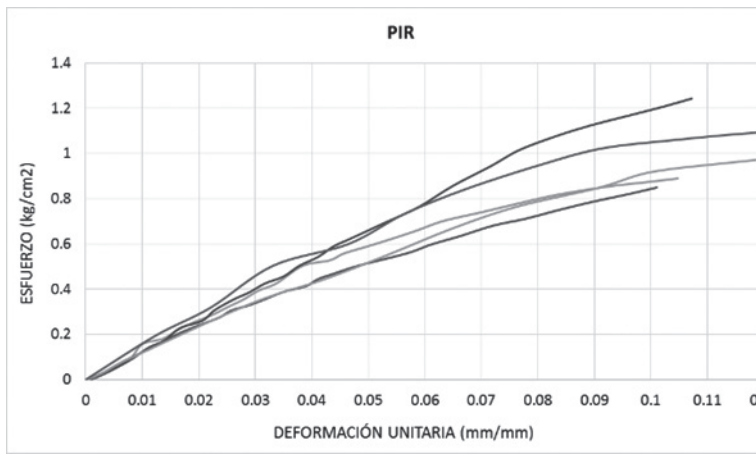

Gráfico 7. Curvas esfuerzo versus deformación unitaria en muestras de PIR.

Ensayo a Compresión Paralela: Este ensayo se realiza utilizando la norma ASTM C364 [9], para determinar el esfuerzo máximo a compresión paralela.
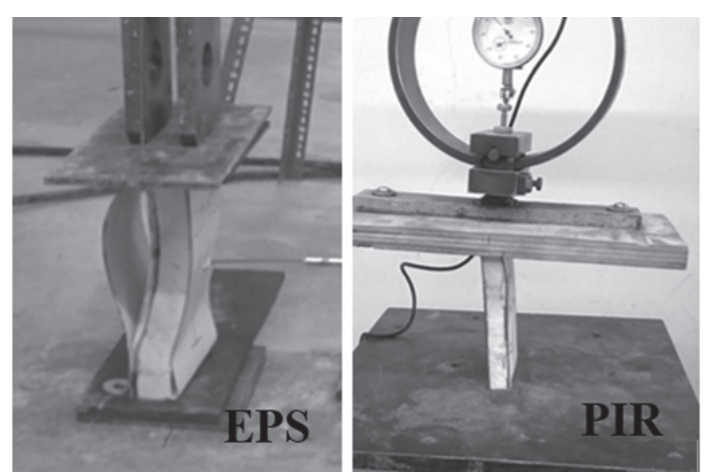

Gráfico 8. Ensayo a compresión paralela. 
Ensayo a Flexión: El ensayo se realiza en los paneles de cubierta (PIR) según la norma ASTM E72 [10], para determinar el esfuerzo máximo a flexión y parámetros de resistencia y rigidez de la sección transversal del panel frente a cargas perpendiculares al plano.
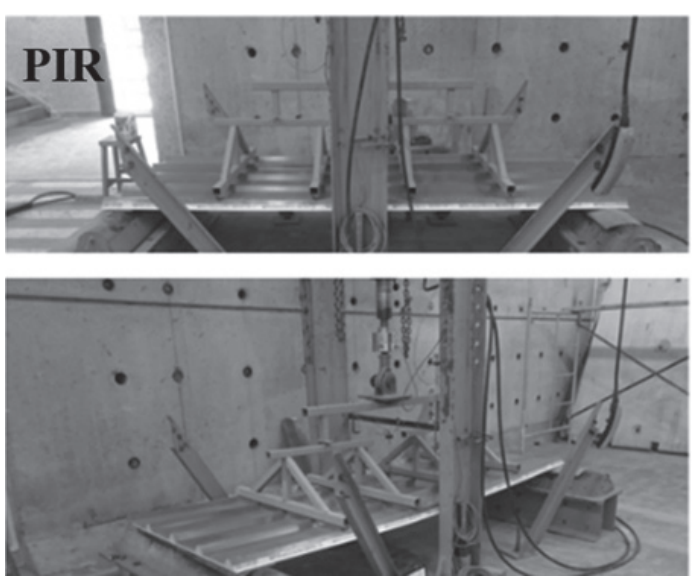

Gráfico 9. Ensayo a flexión.

2.5 Estudio experimental a carga vertical en la vivienda

El estudio a carga vertical evalúa la resistencia y rigidez de la estructura frente a solicitaciones gravitatorias, para ello se realiza una prueba de carga vertical estática en la cubierta y se procesan los datos para determinar deflexiones.

La vivienda de esta investigación posee segmentos idénticos por lo que la prueba de carga se realiza en una sección representativa de la cubierta [11]. La sección de estudio se escoge analizando la zona más crítica, donde las deflexiones y esfuerzos sean máximos.

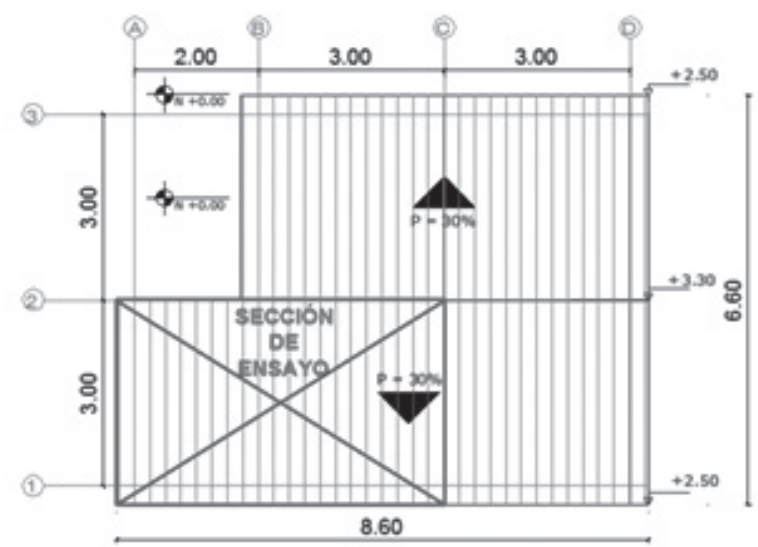

Gráfico 10. Sección de cubierta donde se aplica la sobrecarga.
La carga de estudio para esta vivienda es la carga muerta (peso propio de cubierta) más una sobrecarga de servicio [11]. Para la carga viva se utiliza la indicada para cubiertas inclinadas según la norma NEC-15 [12], y la intensidad de carga aplicada en el ensayo se calcula según el código ACI 318 [11].

\begin{tabular}{l|l|c|c}
\hline \multicolumn{1}{c|}{ TIPO DE CARGA } & \multicolumn{1}{|c|}{ DESCRIPCIÓN } & $\begin{array}{c}\text { CARGA } \\
\mathrm{kPa}\end{array}$ & $\begin{array}{c}\text { CARGA } \\
\mathbf{k g} / \mathrm{m}^{2}\end{array}$ \\
\hline 'CARGA MUERTA & Peso propio panel & 0.068 & 6.886 \\
\hline CARGA VIVA & Carga mínima para cubiertas & 0.700 & 71.380 \\
\hline $\begin{array}{l}\text { INTENSIDAD DE CARGA } \\
\text { REQUERIDA }\end{array}$ & $\begin{array}{l}\text { Carga total de ensayo incluyendo } \\
\text { la carga muerta ya presente }\end{array}$ & 1.092 & 111.338 \\
\hline SOBRECARGA APLICADA & Carga de ensayo & $\mathbf{1 . 0 3 0}$ & $\mathbf{1 0 5 . 0 0 0}$ \\
\hline
\end{tabular}

El proceso de aplicación de carga se realiza en seis etapas, primero se aplica una carga de $0.294 \mathrm{kPa}$ $(30 \mathrm{~kg} / \mathrm{m} 2)$, seguido se realizan incrementos de $0.147 \mathrm{kPa}(15 \mathrm{~kg} / \mathrm{m} 2)$ hasta llegar a la carga de ensayo, y posteriormente se realiza la descarga en un proceso de cinco etapas.

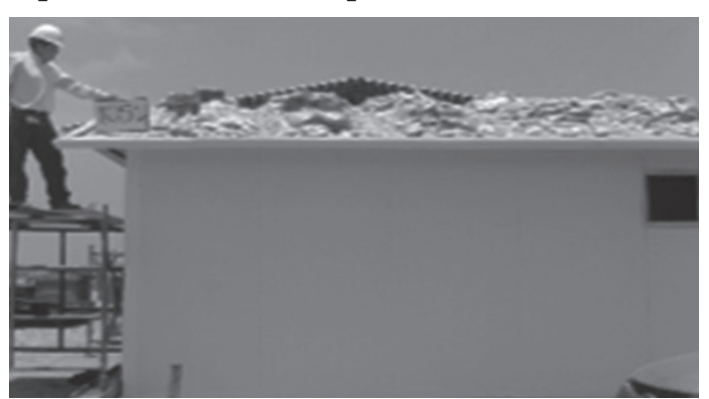

Gráfico 11. Ensayo de prueba de carga vertical.

La instrumentación empleada para registrar las deflexiones son deformímetros mecánicos, mismos que se ubican en el centro de luz de la sección de análisis.

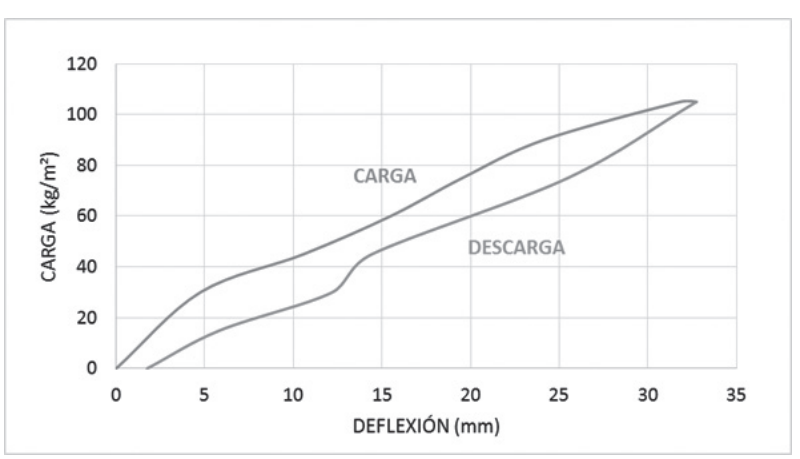

Gráfico 12. Etapas de carga y descarga en prueba de carga vertical. 


\subsection{Estudio experimental a carga lateral en la vivienda}

Mediante el estudio a carga lateral se evalúa la resistencia y estabilidad de la estructura frente a solicitaciones sísmicas, para ello se realiza un ensayo monotónico a carga lateral en el sentido más crítico en la parte superior de los paneles (ver gráfico 14) y se procesan los datos para determinar desplazamientos.

La carga aplicada debe ser superior al cortante basal de la estructura (fuerza demandada por el sismo de diseño). Para el cálculo del cortante basal se utiliza la ecuación y parámetros descritos en la norma NEC-15 [13].

Tabla 2. Valores de carga lateral

\begin{tabular}{|l|c|c|}
\hline \multicolumn{1}{|c|}{ TIPO DE CARGA } & $\begin{array}{c}\text { CARGA } \\
\mathbf{k N}\end{array}$ & $\begin{array}{c}\text { CARGA } \\
\mathbf{k g}\end{array}$ \\
\hline PESO DE LA ESTRUCTURA & 15.602 & 1590.937 \\
\hline CORTANTE BASAL ESTÁTICO & 22.158 & 2259.418 \\
\hline CARGA APLICADA & $\mathbf{2 7 . 4 5 9}$ & $\mathbf{2 8 0 0 . 0 0 0}$ \\
\hline
\end{tabular}

El proceso de aplicación de carga se realiza en dos etapas, en la primera etapa se aplica carga lateral en varias etapas hasta llegar a la carga de ensayo y posteriormente se realiza la descarga. En la segunda etapa para revisar el comportamiento de la estructura se vuelve a cargar a la vivienda hasta una carga determinada en una sola etapa y se descarga para ver su recuperación.

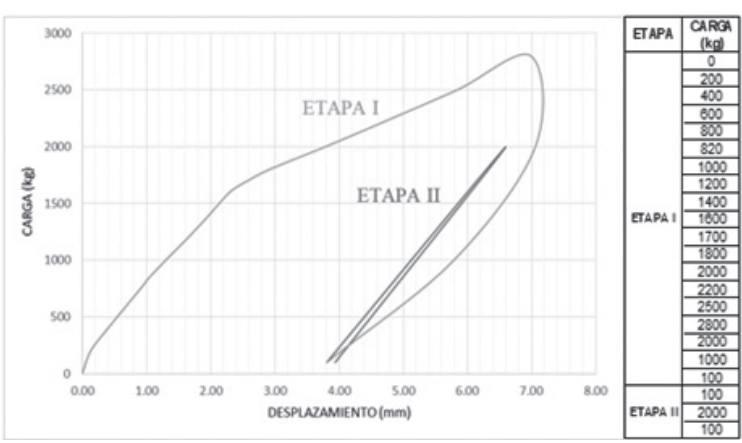

Gráfico 13. Etapas del ensayo a carga lateral.

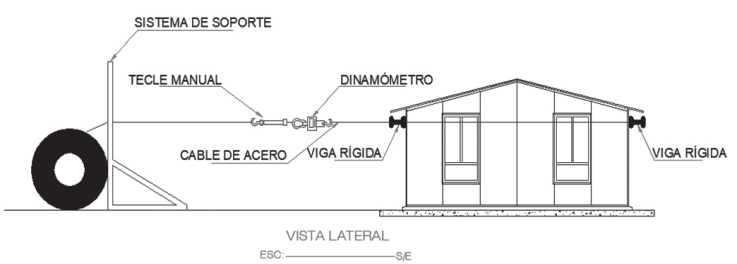

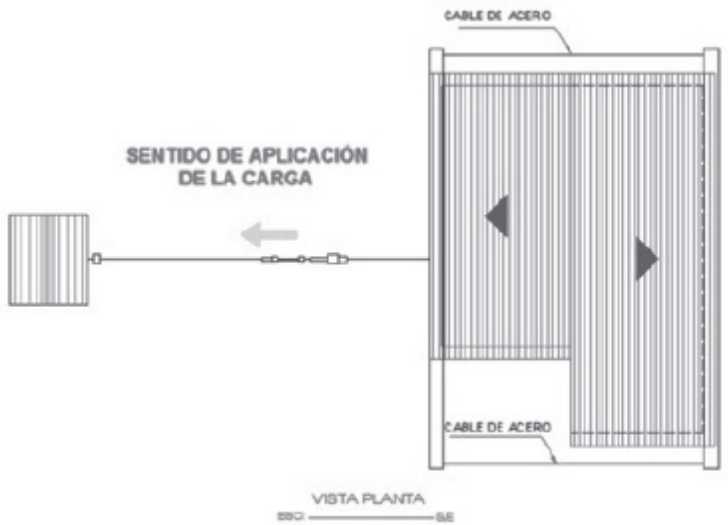

Gráfico 14. Esquema del ensayo a carga lateral.

Se instrumenta el ensayo de carga lateral con tres deformímetros mecánicos, para medir desplazamientos en determinados puntos como se muestra en el gráfico 15.

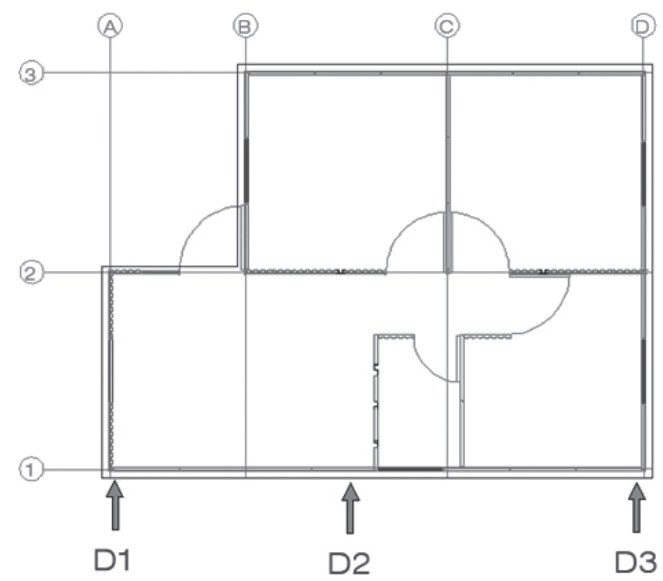

Gráfico 15. Ubicación de medidores de desplazamiento.

\subsection{Medición de vibraciones ambientales en la vivienda}

El estudio de vibraciones ambientales busca determinar el periodo fundamental de la vivienda, si bien este parámetro dinámico de la estructura se puede calcular con modelaciones matemáticas, la medición de vibraciones ambientales da un valor más real del mismo [14].

El equipo utilizado para medir las vibraciones ambientales consistió de un velocímetro-acelerómetro REF-TEK 160-03 marca Trimble, el mismo ha sido provisto por el Instituto Geofísico de la 
Escuela Politécnica Nacional (IG-EPN). Este es un sensor sísmico que permite medir velocidades $y$ aceleraciones en función del tiempo.

Los datos obtenidos de los sensores son procesados de forma similar a la metodología empleada por Guillier et al. [15].

\subsection{Estudio analítico}

El estudio analítico comprende el desarrollo y análisis de un modelo matemático computacional de la vivienda, con el fin de tener un mayor conocimiento del comportamiento y desempeño de la estructura.

En el modelo matemático se simula los ensayos realizados experimentalmente para comprobar que el modelo refleja un comportamiento real de la estructura y conocer los esfuerzos existentes en los diferentes materiales.

Se utilizan dos tipos de elementos para formar los paneles tipo sándwich, se utiliza elementos tipo "Shell" para representar las laminar de acero, y elementos tipo "Solid" para definir el material aislante.
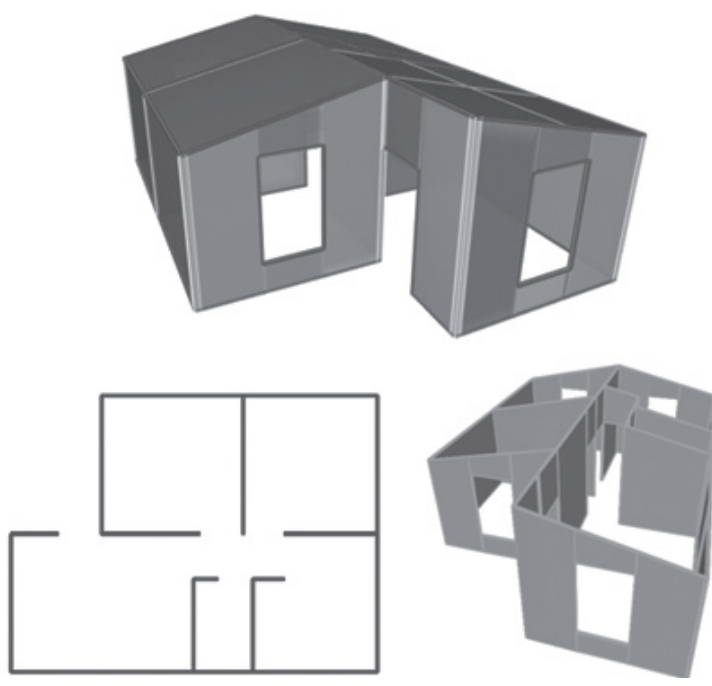

Gráfico 16. Vistas del modelo.

Con el modelo calibrado se obtuvieron esfuerzos críticos en los diferentes elementos para los diferentes estados de carga que se analizaron en los estudios experimentales.

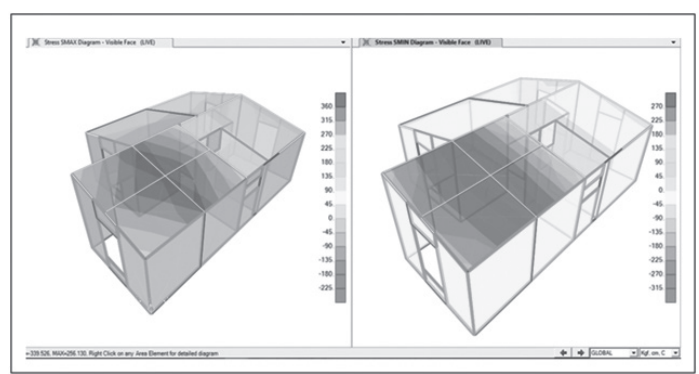

(a)

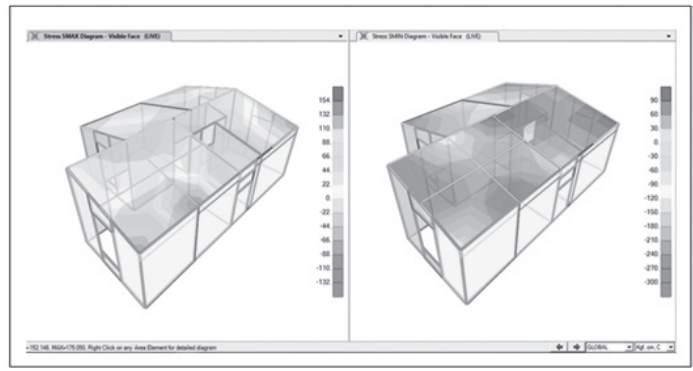

(b)

Gráfico 17. Esfuerzos normales máximos y mínimos para elementos tipo Shell (a) condiciones de carga vertical (b) condiciones de carga lateral.

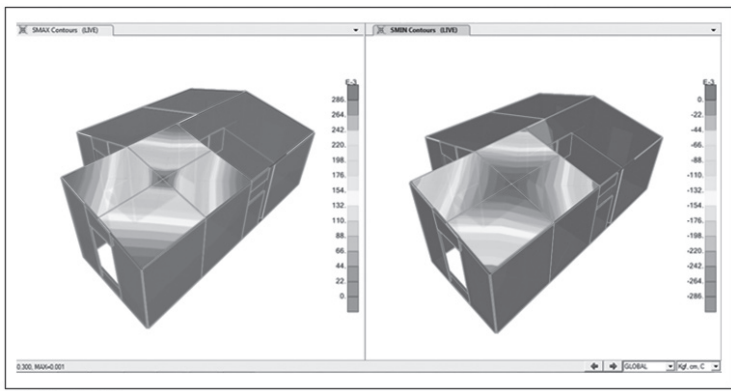

(a)

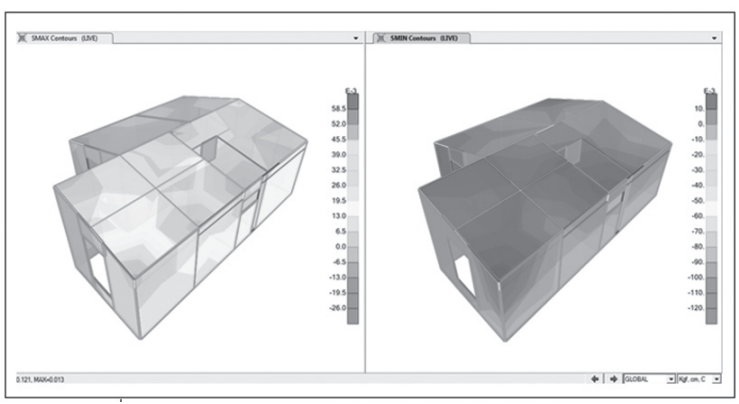

(b)

Gráfico 18. Esfuerzos normales máximos y mínimos para elementos tipo Solid (a) condiciones de carga vertical. (b) condiciones de carga lateral. 


\section{Resultados y discusión}

\subsection{Propiedades físico-mecánicas de los materiales}

En este punto se muestra un resumen de las propiedades obtenidas de los materiales ensayados experimentalmente y estudiados analíticamente.

Tabla 3. Propiedades físico-mecánicas de los materiales

\begin{tabular}{|l|c|c|c|c|c|c|}
\hline $\begin{array}{l}\text { PROPIEDADES FisICO- } \\
\text { MECÁNICAS }\end{array}$ & NORMA & UNIDADES & $\begin{array}{c}\text { NÚCLEO } \\
\text { EPS }\end{array}$ & $\begin{array}{c}\text { NÚCLEO } \\
\text { PIR }\end{array}$ & $\begin{array}{c}\text { PANEL DE } \\
\text { EPS }\end{array}$ & $\begin{array}{c}\text { PANEL DE } \\
\text { PIR }\end{array}$ \\
\hline Densidad & ASTM C271 & $\mathrm{kN} / \mathrm{m}^{3}$ & 0.186 & 0.382 & - & - \\
\hline Esfuerzo a Corte & ASTM C393 & $\mathrm{kPa}$ & 61.880 & 321.853 & - & - \\
\hline $\begin{array}{l}\text { Esfuerzo a Com presión } \\
\text { Perpendicular }\end{array}$ & ASTM C365 & $\mathrm{kPa}$ & 15.396 & 28.243 & - & - \\
\hline $\begin{array}{l}\text { Módulo de Elasticidad } \\
\text { a Compresión }\end{array}$ & ASTM C365 & $\mathrm{kPa}$ & 755.109 & 1192.092 & - & - \\
\hline $\begin{array}{l}\text { Esfuerzo a Com presión } \\
\text { Paralela }\end{array}$ & ASTM C364 & $\mathrm{kPa}$ & 265.563 & 176.225 & - & - \\
\hline Esfuerzo a Flexión & ASTM E72 & $\mathrm{kPa}$ & - & - & 46707.233 & 87639.646 \\
\hline $\begin{array}{l}\text { Módulo de Elasticidad } \\
\text { a Flexión }\end{array}$ & ASTM E72 & $\mathrm{MPa}$ & - & - & 101332.837 & 86150.390 \\
\hline
\end{tabular}

Tabla 4. Resultado de esfuerzos críticos en elementos tipo Shell y elementos tipo Solid

\begin{tabular}{|l|c|c|c|}
\hline \multirow{2}{*}{\multicolumn{1}{|c|}{ ESFUERZO }} & \multicolumn{2}{c|}{ SOLID } & SHELL \\
\cline { 2 - 4 } & $\begin{array}{c}\text { QS } \\
\mathrm{kPa}\end{array}$ & $\begin{array}{c}\text { PIR } \\
\mathrm{kPa}\end{array}$ & $\begin{array}{c}\text { LÁM INA DE ACERO } \\
\mathrm{kPa}\end{array}$ \\
\hline Corte & 1.667 & 6.276 & - \\
\hline Compresión Perpendicular & 2.354 & 29.420 & - \\
\hline Flexión - Tracción & - & - & 34341.780 \\
\hline Flexión - Compresión & - & - & 31674.381 \\
\hline
\end{tabular}

\subsection{Deflexiones obtenidas en carga vertical.}

Para evaluar la deflexión máxima permitida a carga vertical de la vivienda se analizan dos casos: en el primer caso se revisa la deflexión permanente permitida de acuerdo al informe técnico de la UEAtc [16] para paneles de cubierta de poliisocianurato cuya deformación permanente debe ser inferior al límite admitido equivalente a 1/200 de la luz entre ejes. Como segundo punto se analizó la deflexión máxima y la deflexión permanente requerida en una prueba de carga en losas de hormigón armado, de acuerdo al código ACI-318.
Tabla 5. Comparación de deflexiones teóricas y experimentales

\begin{tabular}{|l|c|l|}
\hline \multicolumn{1}{|c|}{ TIPO DEDELEXIÓN } & $\begin{array}{c}\text { DELEXIÓN } \\
\mathrm{mm}\end{array}$ & DESCRIPCIÓN \\
\hline Deflexión permanente permitida & 15.00 & UEAtc \\
\hline Deflexión permanente permitida & 7.50 & $\mathrm{ACI} 318$ \\
\hline Deflexión permantente obtenida & 2.24 & Ensayo \\
\hline Deflexión máxima permitida & 30.00 & ACl 318 \\
\hline Deflexión máxima obtenida & 31.83 & Ensayo \\
\hline
\end{tabular}

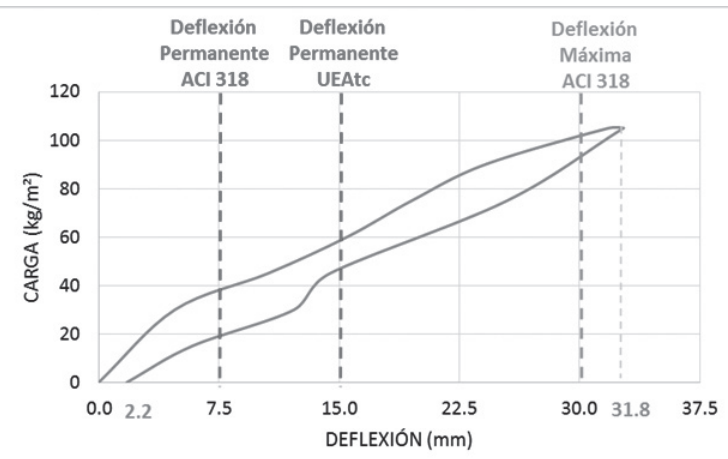

Gráfico 19. Curva carga versus deflexión.

\subsection{Fuerza lateral alcanzada}

De acuerdo a [13], se requiere que la resistencia de la vivienda a carga lateral sea mayor a la demandada por el sismo de diseño (sismo con un periodo de retorno de 475 años). Para ello se determina el cortante basal elástico de la estructura utilizando (1).

$$
V=\frac{I . S a}{R . \emptyset e . \emptyset p} W
$$

Donde:

$$
\begin{array}{ll}
\mathrm{V} & \text { : cortante basal total } \\
\mathrm{I} & : \text { coeficiente de importancia } \\
\mathrm{Sa} & : \text { espectro de diseño en aceleración } \\
\emptyset_{\mathrm{p}} \text { y } & \emptyset_{\mathrm{e}} \text { : coeficientes de configuración en planta } \\
& \text { y elevación } \\
\mathrm{R} & \text { : factor de reducción de resistencia sísmica } \\
\mathrm{W} & \text { : carga sísmica reactiva }
\end{array}
$$


Tabla 6. Comparación de cargas laterales máximas teóricas y experimentales

\begin{tabular}{|l|c|c|l|}
\hline \multicolumn{1}{|c|}{ TIPO DE CARGA } & $\begin{array}{c}\text { CARGA } \\
\mathbf{k N}\end{array}$ & $\begin{array}{c}\text { CARGA } \\
\mathbf{k g}\end{array}$ & \multicolumn{1}{|c|}{ OBSERVACIONES } \\
\hline Peso de la estructura & 15.602 & 1590.937 & W= Peso de la estructura \\
\hline $\begin{array}{l}\text { Cortante basal } \\
\text { estático }\end{array}$ & 22.158 & 2259.418 & $\begin{array}{l}\text { Fuerza demandada por el sismo } \\
\text { de diseño. (equivalente a 1.42 W ) }\end{array}$ \\
\hline Carga aplicada & 27.459 & 2800.000 & $\begin{array}{l}\text { Fuerza de ensayo (equivalente a } \\
1.76 \mathrm{~W} \text { ) }\end{array}$ \\
\hline
\end{tabular}

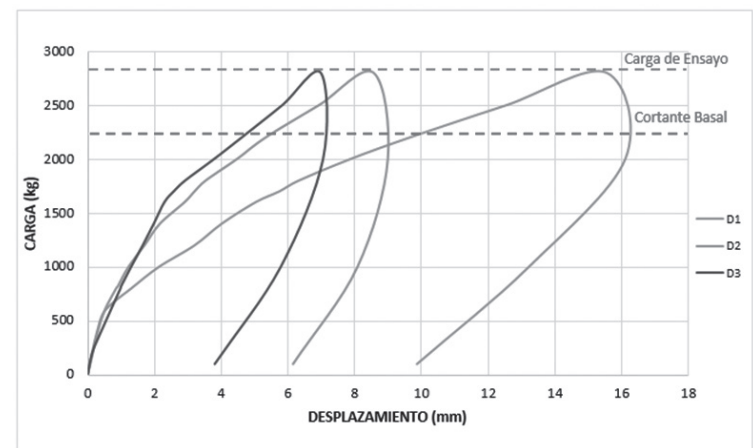

Gráfico 20. Comparación de cargas laterales alcanzadas con las requeridas por el código.

\subsection{Derivas máximas obtenidas en carga lateral.}

En la NEC-15 [13], se requiere que la deriva máxima no exceda el límite de deriva inelástica establecida para los diferentes tipos de estructuras. Debido a que el sistema constructivo de la vivienda no se encuentra descrito en la norma se toma el límite establecido para estructuras de hormigón armado, estructuras metálicas y de madera $(\Delta$ máx $=0.02)$.

Tabla 7. Comparación de derivas máximas teóricas y experimentales

\begin{tabular}{|l|c|}
\hline $\begin{array}{l}\text { Desplazamiento máximo permitida por la norma } \\
(\mathrm{mm})\end{array}$ & 48.000 \\
\hline Deriva máxima permitida por la norma & 0.020 \\
\hline $\begin{array}{l}\text { Desplazamiento máximo obtenido en el ensayo } \\
(\mathrm{mm})\end{array}$ & 9.650 \\
\hline Deriva máxima obtenida en el ensayo & 0.004 \\
\hline $\begin{array}{l}\text { Desplazamiento máximo obtenido en el ensayo } \\
(\mathrm{mm})\end{array}$ & 16.100 \\
\hline Deriva máxima obtenida en el ensayo & 0.007 \\
\hline
\end{tabular}

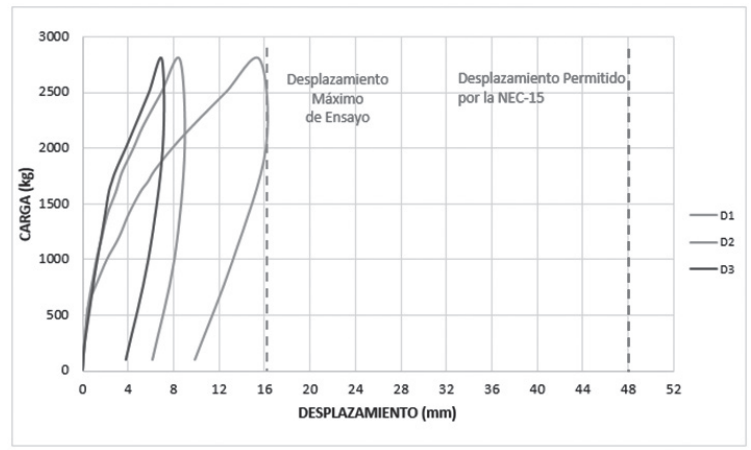

Gráfico 21. Comparación de derivas alcanzadas con las requeridas por el código.

\subsection{Variación de la rigidez}

En el ensayo a carga lateral se aplica carga en dos etapas con el fin de comprobar si la vivienda pierde rigidez durante el ensayo. Para ello se analiza las pendientes de las curvas de capacidad, tanto para la Etapa I como para la Etapa II.

Tabla 8. Comparación de rigidez inicial y rigidez final

\begin{tabular}{|c|c|c|c|}
\hline $\begin{array}{c}\text { RIGIDEZ } \\
\text { kN/m }\end{array}$ & D1 & D2 & D3 \\
\hline $\begin{array}{c}\text { ETAPA I } \\
\text { (ki) }\end{array}$ & 4604.134 & 8038.366 & 7543.697 \\
\hline $\begin{array}{c}\text { ETAPA II } \\
\text { (kf) }\end{array}$ & 4729.170 & 7731.507 & 6678.470 \\
\hline
\end{tabular}

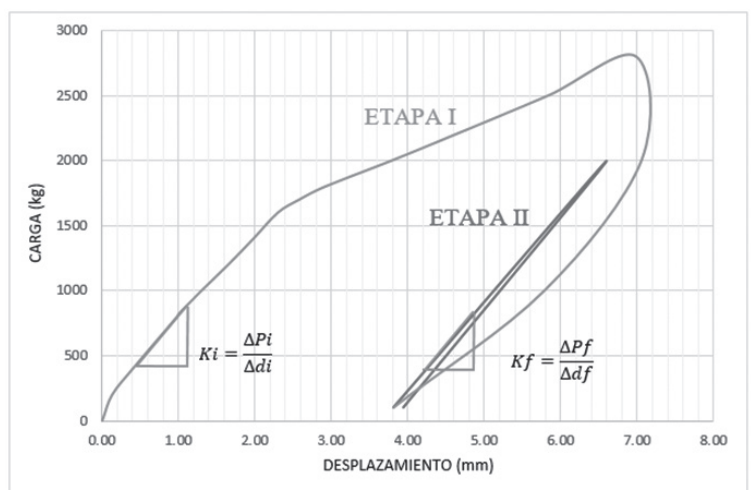

Gráfico 22. Curva carga versus deformación para carga lateral (D3), variación de la rigidez. 


\subsection{Volcamiento}

La vivienda no debe presentar volcamiento para la fuerza demandada por el sismo de diseño.

Para revisar que no exista volcamiento se verifica que el momento resistente de la vivienda sea mayor que el momento volcante.

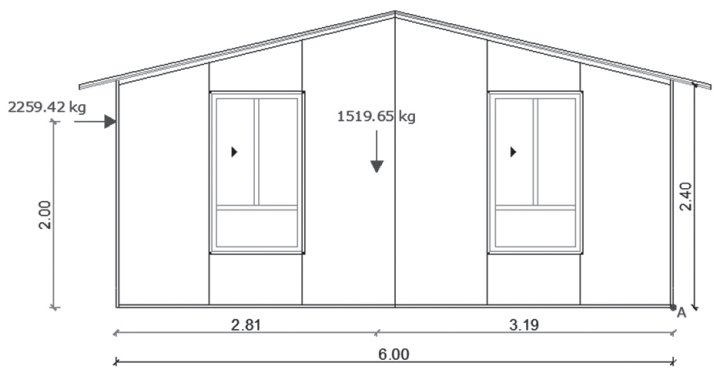

Gráfico 23. Fuerzas resistente y volcante de la vivienda.

Tabla 9. Comparación de momento resistente y momento volcante

\begin{tabular}{|c|c|c|c|c|c|}
\hline & $\begin{array}{l}\text { FUERZA } \\
\qquad \mathrm{kN}\end{array}$ & \begin{tabular}{|c|} 
BRAZO DE \\
APLICACIÓN \\
DE LA FUERZA \\
$\mathbf{m}$
\end{tabular} & $\begin{array}{c}\text { MOMENTO } \\
\text { kN.m }\end{array}$ & $\begin{array}{c}\text { MOMENTO } \\
\text { kg.m }\end{array}$ & DESCRIPCIÓN \\
\hline Resistente & 14.90 & 3.19 & 47.54 & 4847.69 & $\begin{array}{l}\text { Momento que genera el } \\
\text { peso propio }\end{array}$ \\
\hline Volcante & 22.16 & 2.00 & 44.32 & 4518.84 & $\begin{array}{l}\text { Momento que genera el } \\
\text { cortante basal }\end{array}$ \\
\hline
\end{tabular}

3.6 Daños visuales en prueba de carga vertical y ensayo a carga lateral.

Durante la prueba de carga vertical y el ensayo a carga lateral la estructura no presenta signos de desintegración, es decir los paneles nunca se salieron de sus guías metálicas, las uniones no fallan y no se pierde la conexión entre las paredes y la cubierta. Tampoco presenta deformaciones permanentes representativas.

\subsection{Periodo fundamental}

Con las vibraciones ambientales se tuvieron las siguientes señales, mismas que se procesaron para obtener las frecuencias fundamentales en ambas direcciones.
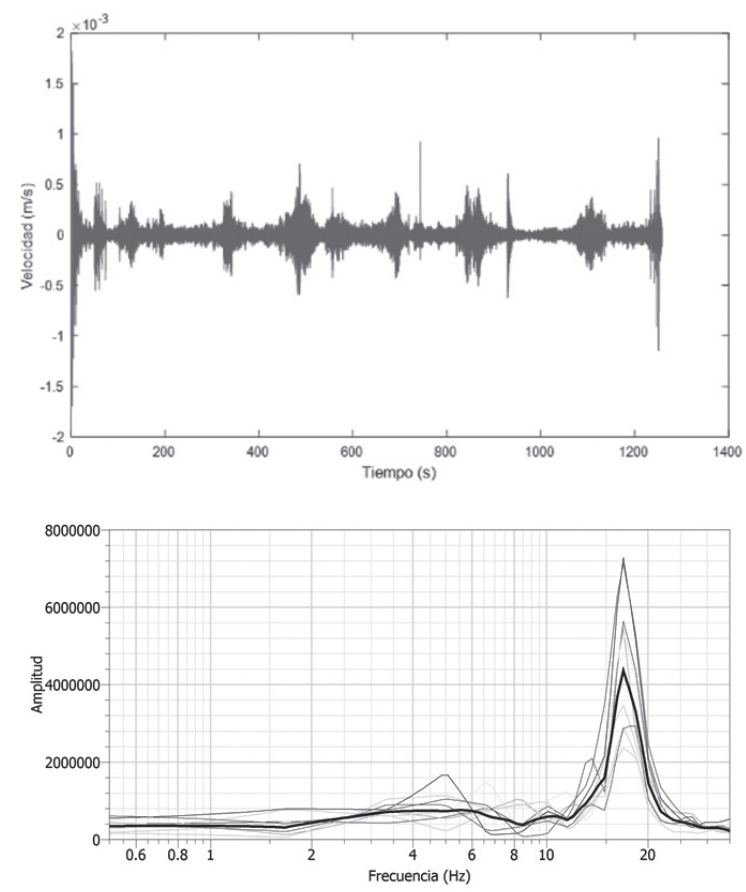

Gráfico 24. Señal y espectro de respuesta obtenido en sentido norte-sur.
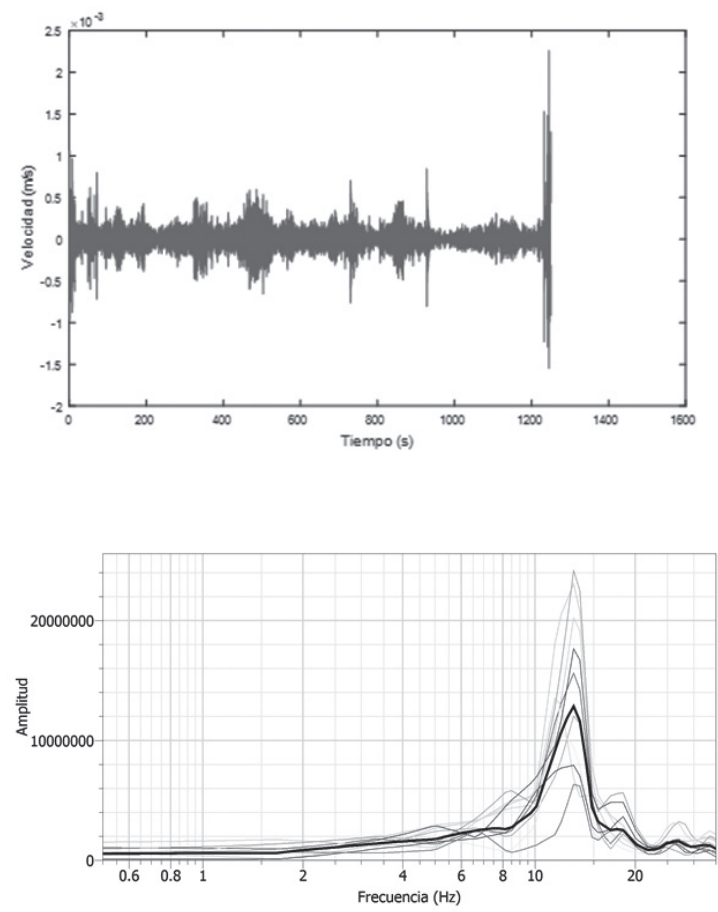

Gráfico 25. Señal y espectro de respuesta obtenido en sentido esteoeste. 
Tabla 10. Resultado de vibraciones ambientales

\begin{tabular}{|c|c|c|}
\hline DIRECCIÓN & $\begin{array}{c}\text { RRECUENCIA } \\
\text { FUNDAMENTAL } \\
(\mathbf{H z})\end{array}$ & $\begin{array}{c}\text { PERIODO } \\
\text { FUNDAMENTAL } \\
\mathbf{( s )}\end{array}$ \\
\hline NORTE-SUR & 17.5323 & 0.0570 \\
\hline ESTE-OESTE & 12.7791 & 0.0783 \\
\hline
\end{tabular}

De la tabla 10 se puede observar que el periodo fundamental de la vivienda es de 0.0783 en el sentido este-oeste (sentido corto de la vivienda).

\subsection{Esfuerzos de corte en espumas de EPS y PIR}

Para revisar si el comportamiento de las espumas de PIR y EPS es el adecuado frente a esfuerzos de corte, se compara el esfuerzo resistente a corte obtenido en el estudio de materiales, con el esfuerzo máximo a corte obtenido del modelo calibrado en los elementos tipo Solid para los diferentes materiales.

Tabla 11. Comparación de esfuerzos de corte obtenidos analítica y experimentalmente

\begin{tabular}{|c|c|c|c|}
\hline DETALLE & $\begin{array}{c}\text { EPS } \\
\mathrm{kPa}\end{array}$ & $\begin{array}{c}\text { PIR } \\
\mathrm{kPa}\end{array}$ & DESGRIPGIÓN \\
\hline ENSAYO DE LABORATORIO & 61.880 & 321.853 & CAPACIDAD \\
\hline MODELO ANALÍTICO CALIBRADO & 1.667 & 6.276 & DEMANDA \\
\hline
\end{tabular}

\subsection{Esfuerzos de compresión en espumas de EPS y} PIR

La revisión del comportamiento de las espumas frente a esfuerzos de compresión, consiste en comparar el esfuerzo resistente a compresión perpendicular obtenido en el estudio de materiales, con el esfuerzo máximo a compresión del modelo calibrado.

Tabla 12. Comparación de esfuerzos de compresión obtenidos analítica y experimentalmente

\begin{tabular}{|c|c|c|c|}
\hline DETALLE & $\begin{array}{c}\text { EPS } \\
\mathrm{kPa}\end{array}$ & $\begin{array}{c}\text { PIR } \\
\mathrm{kPa}\end{array}$ & DESCRIPCIÓN \\
\hline ENSAYO DE LABORATORIO & 15.396 & 28.243 & CAPACIDAD \\
\hline MODEO ANALITICO CALIBRADO & 2.354 & 29.420 & DEMANDA \\
\hline
\end{tabular}

\subsection{Esfuerzos de flexión en láminas de acero}

La revisión del comportamiento de las láminas de acero frente a esfuerzos de flexión, compara el esfuerzo resistente a flexión obtenido en el es- tudio de materiales con los esfuerzos máximos a tracción y compresión obtenidos del modelo calibrado en los elementos tipo Shell.

Tabla 13. Comparación de esfuerzos a flexión obtenidos analítica y experimentalmente.

\begin{tabular}{|c|c|c|c|}
\hline DETALLE & $\begin{array}{c}\text { TRACCIÓN } \\
\mathrm{kPa}\end{array}$ & $\begin{array}{c}\text { COMPRESIÓN } \\
\mathrm{kPa}\end{array}$ & DESCRIPCIÓN \\
\hline ENSAYO DE LABORATORIO & 87639.744 & 87639.744 & CAPACIDAD \\
\hline MODELO ANALITICO CALIBRADO & 34341.780 & 31674.381 & DEMANDA \\
\hline
\end{tabular}

\section{Conclusiones}

La vivienda de esta investigación con la disposición de paredes mostrada tiene una respuesta óptima ante cargas verticales y laterales que representan las acciones sísmicas. Su desempeño la convierte en una vivienda apta para zonas de alto riesgo sísmico.

Las deflexiones obtenidas para una carga de servicio $1.03 \mathrm{kPa}\left(105 \mathrm{~kg} / \mathrm{m}^{2}\right)$ son menores que las deflexiones admisibles recomendadas por el código ACI-318 y el informe técnico emitido por la UEAtc. Por lo que se concluye que la vivienda de estudio cumple con este criterio de aceptabilidad.

La demanda por el sismo de diseño según la NEC$15,22.158 \mathrm{kN}(2259.42 \mathrm{~kg})$ que equivale a $1.42 \mathrm{~W}$ (siendo $\mathrm{W}$ el peso de la estructura) es menor que la resistencia lateral de la estructura $27.459 \mathrm{kN}$ $(2800 \mathrm{~kg})$ que equivale a $1.76 \mathrm{~W}$ en la dirección en que se aplicó la carga. La vivienda cumple con este criterio de aceptabilidad.

La deriva máxima obtenida (0.0067) en dirección de la aplicación de la carga lateral, no excede el límite de deriva inelástica (0.02) establecida en la norma NEC-15 [13]. Se puede concluir que la vivienda cumple con este criterio de aceptabilidad para la dirección en que se realizó el ensayo.

En el ensayo a carga lateral, al incrementar las fuerzas el comportamiento de la estructura se mantuvo en el rango elástico durante todo el ensayo en lo que se refiere a los elementos, pero se observa un comportamiento no lineal de la estructura al sobrepasar los $17.65 \mathrm{kN}$ (1800 kg) aproximadamente, debido al rozamiento entre paneles y conexiones existentes. Debido a que la vivienda resistió la fuerza demandada por el sis- 
mo de diseño en el rango elástico, se concluye que la vivienda tiene un comportamiento aceptable bajo cargas laterales.

Luego de realizar los ensayos a carga vertical y carga lateral, no se encontraron daños visuales en la estructura, esto se comprobó con el monitoreo de fisuras en los testigos de yeso realizado en el ensayo a carga lateral, en el cual se presentan fisuras pequeñas no generalizadas para las cargas a las que fue sometida la vivienda. Es decir, la vivienda cumple con este criterio de aceptabilidad.

Del análisis de esfuerzos realizado se concluye que los esfuerzos actuantes en los materiales núcleo de la vivienda (espuma de EPS y PIR), tanto para cargas verticales como para cargas laterales no exceden a los esfuerzos últimos resistentes de los materiales, por lo que se comprueba que la vivienda tuvo un buen desempeño a nivel de esfuerzos ya que no presento fallas por corte o compresión en las espumas de cubierta ni de pared durante los ensayos experimentales.

En caso de existir algún cambio en la disposición de paredes en la vivienda, se recomienda utilizar el modelo calibrado puesto que refleja un comportamiento cercano a la realidad.

Es importante realizar más ensayos de laboratorio en los paneles utilizados en las paredes, para tener un mayor conocimiento de su desempeño frente a cargas laterales.

Se sugiere cambiar el material de relleno de las paredes (espuma de EPS) que, si bien tuvo un buen desempeño durante los ensayos a carga lateral y vertical, este material no presentó una buena resistencia al calor al acondicionarlo para el cálculo de la densidad (ensayo de densidad), ya que el material se quemó y redujo su sección a la mitad.

\section{Reconocimiento}

Gracias al personal del Centro de Investigación de la Vivienda de la Escuela Politécnica Nacional por su colaboración durante la ejecución de esta investigación.

Gracias a la empresa KUBIEC por su contribución durante la realización de esta investigación.

\section{Referencias}

[1] M. Iza, Modelación de un sistema estructural alternativo, prefabricado de bajo costo, para una vivienda de dos pisos. (Paneles de metálicos con inyección de poliuretano de alta densidad), Quito, Pichincha, 2012, p. 200.

[2] C. Abeysinghe, D. Thambiratnam and N. PererA, Flexural performance of an innovative Hybrid Composite Floor Plate System, 2013, p. 26.

[3] D. Bournas, G. Torrisi, F. Crisafulli and A. Pavese, Experimental Investigation and Analytical Modeling of Prefabricated Reinforced Concrete Sandwich Panels, vol. 14, Portugal, 2012.

[4] NEC-15 Capítulo 9, NEC-SE-VIVIENDA: Viviendas de hasta 2 pisos con luces de hasta 5 metros, 2015.

[5] KUBIEC, KUTÉRMICO paneles tipo sánduche con aislamiento termoacústico, Quito, Pichincha, 2015.

[6] ASTM C271, Standard Test Method for Density of Sandwich Core Materials., ASTM, 2017.

[7] ASTM C393, Standart Test Method for Core Shear Properties of Sandwich Constructions by Beam., ASTM, 2017.

[8] ASTM C365, Standart Test Method for Flatwise Compressive Strength of Sandwich Constructions, ASTM, 2017.

[9] ASTM C364, Standart Test Method for Edgewise Compressive Strength of Sandwich Constructions, ASTM, 2017.

[10] ASTM E72, Standart Test Method of Conducting Strength Tests of Panels for Building Constructions, ASTM, 2017.

[11] A. C. I. ACI 318S, Requisitos de Reglamento para Concreto Estructural y Comentario, Estados Unidos, 2014.

[12] NEC-15 Capítulo 1, NEC-SE-CG: Cargas no sísmicas, 2015.

[13] NEC-15 Capítulo 2, NEC-SE-DS: Peligro sísmico y requisitos de diseño sismo resistente, 2015. 
[14] G. Parra and J. Sarango, Análisis estadístico del periodo experimental de vibración de edificios aporticados de hormigón armado en el Distrito Metropolitano de Quito. Quito, Pichincha, 2016.

[15] B. Guillier, J. L. Chatelain, H. Tavera, H. Perfettini, A. Ochoa and B. Herrera, Establishing Empirical Period Formula for RC Buildings in Lima, Peru: Evidence for the Impact of Both the 1974 Lima Earthquake and the Application of the Peruvian Seismic Code on High-Rise Buildings., vol. 85(6), Seismological Research Letters, 2014, pp. 1308-1315.
[16] Unión Europea para la Idoneidad Técnica en la Construcción, "Informe técnico de la UEAtc para la evaluación de las obras realizadas con paneles sándwich de espuma de poliuretano sin CFC", 1996. 